\title{
PepHMM: A Hidden Markov Model Based Scoring Function for Mass Spectrometry Database Search
}

\author{
Yunhu Wan, Austin Yang, and Ting Chen
}

\section{Support Information}

\section{Dynamic Programming Algorithm for HMM}

We first introduce the likelihood model of the HMM, and then we provide algorithms for computing the likelihood. At the end, we describe how to calculate emission probabilities. The notations are defined as follows. Let $s$ be the given spectrum. Let $p$ be a candidate peptide. Let $N$ be the number of positions (the number of fragmentations for $p$ ) in HMM. We define $M$ as the observations in which we match $p$ with $s$ ( $M$ is the input to the HMM structure). Let $\pi_{i}$ be the state at the $i$-th position.

The parameters of the HMM are defined as follows. Let $a_{u, v}^{(i)}$, be the transitional probability from state $u$ at position $i$ to state $v$ at position $i+1$, and $e_{u}^{(i)}$ be the emission probability of state $u$ at position $i$ (the position $i$ may be omitted if it is clear from the context). The emission probabilities are determined by $\mu$ and $\sigma$ of the normal distribution of the match tolerance and $\lambda_{b}$ and $\lambda_{y}$ of the exponential distribution of the peak intensity for $\mathrm{b}$ ions and $\mathrm{y}$ ions respectively. Define $\theta=\left(a, \mu, \sigma, \lambda_{b}, \lambda_{y}\right)$ to be the parameters of the HMM model. Given $\theta$, we can find the highest-score peptide from a database $D$ for $s$ :

$$
\max _{p \in D} \operatorname{Pr}(s \mid p, \theta) .
$$

Let a path at HMM be $\pi=\pi_{1} \pi_{2} \cdots \pi_{N}$ (or a configuration of states). We add $\pi_{0}$ at the beginning of $\pi$ to indicate the starting state. The probability of this path,

$$
\begin{aligned}
\operatorname{Pr}(\pi, s \mid p, \theta) & =\operatorname{Pr}(\pi, M \mid \theta) \\
& =\prod_{i=0}^{N-1}\left(a_{\pi_{i}, \pi_{i+1}} e_{\pi_{i+1}}\right) * \eta^{\# \text { noise }},
\end{aligned}
$$

where $a_{\pi_{0}, \pi_{1}}$ is the prior probability of the first state $\pi_{1}, a_{\pi_{i}, \pi_{i+1}}$ is the transitional probability from state $\pi_{i}$ to state $\pi_{i+1}, e_{\pi_{i}}$ is the emission probability 
of state $\pi_{i}$, and $\eta$ is the probability of observing a noise peak. Considering all possible paths,

$$
\operatorname{Pr}(s \mid p, \theta)=\operatorname{Pr}(M \mid \theta)=\sum_{\pi} \operatorname{Pr}(\pi, M \mid \theta) .
$$

Because there are an exponential number of possible paths, we can use either the forward algorithm or the backward algorithm to calculate this probability. These two algorithms are also used in estimating the parameters $\theta$.

Forward Algorithm. Define $f_{v}^{(i)}$ as the probability of all possible paths from the first position to state $v$ at position $i$, or

$$
f_{v}^{(i)}=\sum_{\pi_{1}, \ldots, \pi_{i-1}} \operatorname{Pr}\left(\pi_{1}, \ldots, \pi_{i-1}, \pi_{i}=v, M \mid \theta\right) .
$$

$f_{v}^{(i)}$ can be calculated by the following recursion,

$$
f_{v}^{(i)}=e_{v}^{(i)} \sum_{u}\left(f_{u}^{(i-1)} a_{u, v}^{(i-1)}\right)
$$

with an initial value $f_{v}^{(1)}=a_{0, v} e_{v}^{(1)}$. Using a dynamic programming algorithm, we can compute the final probability

$$
\operatorname{Pr}(M \mid \theta)=\sum_{v} f_{v}^{(N)}
$$

Backward Algorithm. Define $b_{u}^{(i)}$ as the probability of all possible paths from state $u$ at position $i$ to any state at the final position, or

$$
b_{u}^{(i)}=\operatorname{Pr}\left(\pi_{i+1}, \cdots, \pi_{N}, \pi_{i}=u, M \mid \theta\right) .
$$

Similarly, $b_{u}^{(i)}$ can be calculated by the following recursion,

$$
b_{u}^{(i)}=\sum_{v}\left(a_{u, v}^{(i)} e_{v}^{(i+1)} b_{v}^{(i+1)}\right),
$$

with an initial value $b_{u}^{(N)}=1$. Using a dynamic programming algorithm, we can compute the final probability

$$
\operatorname{Pr}(M \mid \theta)=\sum_{u}\left(a_{0, u} e_{u}^{(1)} b_{u}^{(1)}\right) .
$$

Calculating Emission Probability. The probability of observing $\left(T_{b}, I_{b}\right)$ and $\left(T_{y}, I_{y}\right)$ at state 1 (observation of both $\mathrm{b}$ and $\mathrm{y}$ ions) of position $i$ is

$$
\begin{aligned}
e_{1}^{(i)} & =\operatorname{Pr}\left(\left(T_{b}, I_{b}\right),\left(T_{y}, I_{y}\right) \mid \theta\right) \\
& =\operatorname{Pr}\left(\left(T_{b}, I_{b}\right) \mid \theta\right) \operatorname{Pr}\left(\left(T_{y}, I_{y}\right) \mid \theta\right) \\
& =\operatorname{Pr}\left(T_{b} \mid \theta\right) \operatorname{Pr}\left(I_{b} \mid \theta\right) \operatorname{Pr}\left(T_{y} \mid \theta\right) \operatorname{Pr}\left(I_{y} \mid \theta\right),
\end{aligned}
$$

where $\operatorname{Pr}\left(T_{b} \mid \theta\right)$ and $\operatorname{Pr}\left(T_{y} \mid \theta\right)$ can be calculated by the normal distribution $N(\mu, \sigma)$, and $\operatorname{Pr}\left(I_{b} \mid \theta\right)$ and $\operatorname{Pr}\left(I_{y} \mid \theta\right)$ can be calculated by the exponential distributions with means $\lambda_{b}$ and $\lambda_{y}$ respectively. The emission probabilities of other states can be calculated similarly. 


\section{EM Algorithm}

Assume the training set includes a total of $K$ pairs of peptides and mass spectra, $\left(p_{1}, s_{1}\right), \cdots,\left(p_{K}, s_{K}\right)$. We obtain $K$ observations $M=M_{1}, \cdots, M_{K}$ by matching peptides with spectra. Our goal is to find $\theta$ that maximize the likelihood $l(\theta \mid M)$. Define $\{\gamma, \xi\}$ as missing data, where $\gamma_{v}^{(i)}(k)$ is the probability of state $v$ at position $i$ for the $k$-th spectra given the observation $M_{k}$ and $\theta$, and $\xi_{u, v}^{(i)}(k)$ is the probability of state $u$ at position $i$ and state $v$ at position $i+1$ for the $k$-th spectra given the observation $M_{k}$ and $\theta$. The EM algorithm estimates the expectation of the missing data as follows (E-step):

$$
\begin{aligned}
\gamma_{v}^{(i)}(k) & =\operatorname{Pr}\left(\pi_{i}=v \mid M_{k}, \theta\right) \\
& =\frac{f_{v}^{(i)}(k) b_{v}^{(i)}(k)}{\operatorname{Pr}\left(M_{k} \mid \theta\right)}, \\
\xi_{u, v}^{(i)}(k) & =\operatorname{Pr}\left(\pi_{i}=u, \pi_{i+1}=v \mid M_{k}, \theta\right) \\
& =\frac{f_{u}^{(i)}(k) a_{u, v}^{(i)}(k) e_{v}^{(i+1)}(k) b_{v}^{(i+1)}(k)}{\operatorname{Pr}\left(M_{k} \mid \theta\right)},
\end{aligned}
$$

Using the missing data we compute $\theta$ to maximize the likelihood (M-step). The transitional probability,

$$
\hat{a}_{u v}^{(i)}=\frac{\sum_{k=1}^{K} \xi_{u, v}^{(i)}(k)}{\sum_{k=1}^{K} \gamma_{u}^{(i)}(k)} .
$$

Let $\left(T_{b}^{(i)}(k), I_{b}^{(i)}(k)\right)$ and $\left(T_{y}^{(i)}(k), I_{y}^{(i)}(k)\right)$ be the observations of the $\mathrm{b}$ ion and $\mathrm{y}$ ion matches at the $i$-th fragmentation (or position at HMM) for the $k$-th peptide. The parameters for the normal distribution of the match tolerance,

$$
\begin{gathered}
\mu=\frac{\sum_{k=1}^{K} \sum_{i=1}^{N}\left(\gamma_{1}^{(i)}(k)+\gamma_{2}^{(i)}(k)\right) T_{b}^{(i)}(k)+\left(\gamma_{1}^{(i)}(k)+\gamma_{3}^{(i)}(k)\right) T_{y}^{(i)}(k)}{\sum_{k=1}^{K} \sum_{i=1}^{N}\left(2 \gamma_{1}^{(i)}(k)+\gamma_{2}^{(i)}(k)+\gamma_{3}^{(i)}(k)\right)} \\
\sigma^{2}=\frac{\sum_{k=1}^{K} \sum_{i=1}^{N}\left(\gamma_{1}^{(i)}(k)+\gamma_{2}^{(i)}(k)\right)\left(T_{b}^{(i)}(k)-\mu\right)^{2}+\left(\gamma_{1}^{(i)}(k)+\gamma_{3}^{(i)}(k)\right)\left(T_{y}^{(i)}(k)-\mu\right)^{2}}{\sum_{k=1}^{K} \sum_{i=1}^{N}\left(2 \gamma_{1}^{(i)}(k)+\gamma_{2}^{(i)}(k)+\gamma_{3}^{(i)}(k)\right)} .
\end{gathered}
$$

The parameters for the exponential distribution of the $\mathrm{b}$ ion intensities and the $\mathrm{y}$ ion intensities,

$$
\begin{aligned}
\lambda_{b} & =\frac{\sum_{k=1}^{K} \sum_{i=1}^{N}\left(\gamma_{1}^{(i)}(k)+\gamma_{2}^{(i)}(k)\right)}{\sum_{k=1}^{K} \sum_{i=1}^{N} I_{b}^{(i)}(k)\left(\gamma_{1}^{(i)}(k)+\gamma_{2}^{(i)}(k)\right)}, \\
\lambda_{y} & =\frac{\sum_{k=1}^{K} \sum_{i=1}^{N}\left(\gamma_{1}^{(i)}(k)+\gamma_{3}^{(i)}(k)\right)}{\sum_{k=1}^{K} \sum_{i=1}^{N} I_{y}^{(i)}(k)\left(\gamma_{1}^{(i)}(k)+\gamma_{3}^{(i)}(k)\right)} .
\end{aligned}
$$

The EM algorithm iterates between the E-step and the M-step until it converges. 